\title{
Explosion of Atom Clusters in a Free-Electron Intense Laser Pulse
}

\author{
M. Rusek AND A. Oreowski \\ Institute of Physics, Polish Academy of Sciences \\ al. Lotników 32/46, 02-668 Warsaw, Poland
}

(Received March 30, 2004)

\begin{abstract}
The explosion of rare-gas atomic clusters induced by short, intense X-ray pulses generated by a free-electron laser is studied. A numerical approach for an explicitly time-dependent description of small to medium size clusters in $3 \mathrm{D}$ is developed within the Thomas-Fermi model. Such an approach, though strongly simplified in comparison to fully quantum-mechanical schemes, is nevertheless expected to yield a qualitatively correct description of the electronic and ionic dynamics of these systems, at a much lower computational cost.
\end{abstract}

PACS numbers: 36.40.-C, 32.80.Rm, 42.50.Hz, 07.05.Tp

\section{Introduction}

Intense X-radiation from free-electron lasers has opened many new research areas in physics and chemistry. The ability to ionize matter allows direct insight into electronic structure and chemical composition. Diffraction of X-rays allows to determine the structure of complex elements with atomic resolution. Free-electron lasers combine the advantages of synchrotron radiation (short wavelength) with those of lasers (intense short pulses) and thus open the way to new types of experiments. Recently, the study of the interaction of intense X-rays with atoms and clusters has been reported [1].

Absorption of short-wavelength radiation and subsequent ionization of clusters differs considerably from that in the optical spectral range. The experimental results give evidence that after many unbound electrons are created, a plasma is formed. Free-electron laser energy absorption in such cluster plasma is stronger than predicted by calculations with classical models. Thus quantum-mechanical 
modeling is needed to explain the efficient energy deposition seen at these short wavelengths.

In this paper we further investigate a theoretical approach to explosion of rare-gas atomic clusters in intense laser pulses based on a time-dependent ThomasFermi model introduced in our previous article [2]. The Thomas-Fermi model is the earliest and the simplest of the density functional methods. It was introduced by [3] and [4] in order to describe the self-consistent potential of multi-electron atoms. The advantage of this model is its simplicity (atomic number $Z$ is the only parameter) and universality (different atoms differ by scaling units only). The time-dependent version of the Thomas-Fermi model which we use in this paper may be considered as a semiclassical approximation (described by a Bloch-like hydrodynamic model) to the rigorous quantum dynamics of an electron gas. Let us begin by recalling the basic assumptions of the model.

The paper is organized as follows. In Sec. 2 a version of the time-dependent Thomas-Fermi model is introduced. Then in Sec. 3 some relevant calculations are carried out. Structure of the modeled clusters as well as properties of the pulses are presented in Sec. 4. Main results following from the model are described in Sec. 5. Finally we finish with a brief summary.

\section{Time-dependent Thomas-Fermi model}

Within the Thomas-Fermi model the ground state structure of a rare-gas atomic (van der Waals) cluster is described by the average electron density $\rho(r)$ and the positions of the nuclei $\boldsymbol{R}_{a}$. It can be obtained by minimization of the following Thomas-Fermi energy functional [2]:

$$
\begin{aligned}
\mathcal{E}\left[\rho(\boldsymbol{r}),\left\{\boldsymbol{R}_{a}\right\}\right] & =\int \mathrm{d}^{3} r \rho(\boldsymbol{r}) U(\boldsymbol{r}) \\
& +\frac{e}{2} \int \mathrm{d}^{3} r\left[\sum_{a=1}^{N} Z \delta\left(\boldsymbol{r}-\boldsymbol{R}_{a}\right)-\rho(\boldsymbol{r})\right] \Phi(\boldsymbol{r}),
\end{aligned}
$$

where the relations for the internal (kinetic) energy of an ideal electron gas at temperature $T=0$ are used locally

$$
U(r)=c_{k}[\rho(r)]^{2 / 3},
$$

and the electrons and nuclei interact through the self-consistent Coulomb potential $\Phi(r):$

$$
\Phi(r)=e \int \frac{\mathrm{d}^{3} r^{\prime}}{\left|\boldsymbol{r}-\boldsymbol{r}^{\prime}\right|}\left[\sum_{a=1}^{N} Z \delta\left(\boldsymbol{r}^{\prime}-\boldsymbol{R}_{a}\right)-\rho\left(\boldsymbol{r}^{\prime}\right)\right] .
$$

Here $N$ is the number of atoms in the cluster and $e$ is the elementary charge. 
To introduce a time-dependent version of the Thomas-Fermi model we assume that the electron density at any point of space is obtained by summing up the contributions from $n$ smoothed pseudo-particles [2]:

$$
\rho(r)=\frac{Z N}{n} \sum_{j=1}^{n} f_{j}\left(r-r_{j}\right) .
$$

The functions $f_{j}$ are assumed to be spherically symmetric $f_{j}(r)=f_{j}(|r|)$, and normalized $\int \mathrm{d}^{3} r f_{j}(r)=1$.

The dynamics of the electron density (4) and the motion of the nuclei is governed by the following Hamiltonian in which the Thomas-Fermi energy functional (1) plays a role of the potential energy term [2]:

$$
\begin{aligned}
& \mathcal{H}\left[\rho(\boldsymbol{r}),\left\{\boldsymbol{R}_{a}\right\}\right]=\sum_{a=1}^{N} \frac{M \boldsymbol{V}_{a}^{2}}{2}+\int \mathrm{d}^{3} r \rho(r) \frac{m[v(\boldsymbol{r})]^{2}}{2} \\
& +\mathcal{E}\left[\rho(\boldsymbol{r}),\left\{\boldsymbol{R}_{a}\right\}\right],
\end{aligned}
$$

where $M$ denotes the nuclear mass.

The resulting Hamilton equations of motion for the smoothed pseudo-particles positions $r_{j}$ can be understood as a discretized smooth particle hydrodynamics $[5,6]$ version of the Bloch-like hydrodynamic equations [7]. Therefore, by treating the Thomas-Fermi energy functional as a potential energy part of the Hamiltonian of the system we had viewed the oscillations of the electron cloud in an atomic cluster as a motion of a fluid characterized by a density $\rho(\boldsymbol{r}, t)$ and a velocity field $v(r, t)$. A similar hydrodynamic model has been used in the weak-field limit to study photoabsorption of an atom in free space [8] and in plasma [9].

The hydrodynamic equations for the electron density $\rho$ are to be supplemented by the Newton-like equations of motion for the positions of the nuclei $\boldsymbol{R}_{a}[2]$

The interaction with the laser pulse is treated within the dipole approximation by replacing the electrostatic Coulomb potential in Eq. (3) by

$$
\Phi(r, t) \rightarrow \Phi(r, t)-r \cdot \boldsymbol{F}(t),
$$

where $\boldsymbol{F}(t)$ is the electric field of the incoming wave. The linearly polarized wave of a pulse used in the simulations is assumed to have a field envelope proportional to sine squared with a full width at half maximum $\tau$ and an optical period $\tau_{0}$ :

$$
\boldsymbol{F}(t)=\boldsymbol{F}_{0} \sin ^{2}\left(\frac{\pi}{2 \tau} t\right) \cos \left(\frac{2 \pi}{\tau_{0}} t\right) .
$$

The pulse used in the simulations had a wavelength $98 \mathrm{~nm}$ (or $\tau_{0}=$ 13.527 a.u.), temporal full width at half maximum of $50 \mathrm{fs}$ ( or $\tau / \tau_{0}=150$ ), and the peak intensity of $10^{14} \mathrm{~W} / \mathrm{cm}^{2}$ (or $\left|\boldsymbol{F}_{0}\right|=0.05$ a.u.). These parameters were used in the experiments by Wabnitz [1]. The energy of a single photon is $12.7 \mathrm{eV}$ (or 0.46 a.u.). 


\section{Details of numerical calculations}

In our previous paper [2] we utilized $f_{j}$ from Eq. (4) in the form of a Gaussian function identical for all pseudo-particles

$$
f(\boldsymbol{r})=\left(\frac{\alpha}{\sqrt{\pi}}\right)^{3} \mathrm{e}^{-(\alpha \boldsymbol{r})^{2}}
$$

It turned out, however, that this choice of fixed width of all pseudo-particles leads to some problems in modeling the electron cloud close to the nucleus. Several pseudo-particles were stacking atop on each other and the smooth particle hydrodynamic method was breaking down. To prevent this we have chosen a large value of the parameter $\alpha(\alpha=7$ for $Z=18$ and $n / N=125)$ from Eq. (8) which in turn made the numerical code extremely slow and turned any realistic simulations nearly impossible. Therefore a quite complicated code with variable test-particle width seems to be an obvious choice at a first glance.

On the other hand, however, our experiments with a simpler constant width code showed that in the case of moderate-intensity short-wave laser pulse used in the experiments by Wabnitz [1], the dynamics of core electrons does not play any role in the laser energy absorption process. Therefore instead of modeling the electron cloud close to the nucleus with a large number of test-particles of very small width we made a more physical choice - the electron density of an atom is modeled by test-particles of two kinds: one large particle originally centered at the nucleus which represents the core electrons and a large number of smaller particles modeling the outer shells of an atom

$$
\rho(r)=\frac{Z N}{n}\left\{\xi\left[\sum_{j=1}^{n} f\left(r-r_{j}\right)\right]+(1-\xi) \frac{n}{N} g\left(r-r_{0}\right)\right\}
$$

where $f$ is given by Eq. (8) and

$$
g(r)=\left(\frac{\beta}{\sqrt{\pi}}\right)^{3} \mathrm{e}^{-(\beta \boldsymbol{r})^{2}} .
$$

We have chosen $\beta=1 / r_{0}$ where $r_{0}$ is the radius of the Thomas-Fermi ion of charge $Z(1-\xi)$ (i.e., $Z \xi$ electrons remain to be bound) [10]:

$$
r_{0}=3.17361 \frac{\sqrt{(1-\xi) / \xi}}{Z^{1 / 3}} \text {. }
$$

The parameter $\alpha$ from Eq. (8) was chosen to model an isolated Thomas-Fermi atom of given $Z$ [2]. At this value of $\alpha$ the Thomas-Fermi energy relations are preserved, i.e., $2 \mathcal{E}_{\text {int }}+\mathcal{E}_{\text {pot }}=0$ [11] where $\mathcal{E}_{\text {int }}$ is the internal energy of the electron gas [first term in Eq. (1)] and $\mathcal{E}_{\text {pot }}$ is the potential energy of the electron gas [second term in Eq. (1)]. For example for $n / N=125$ pseudo-particles per atom (this is the value used later in the paper) and $Z=18$ (argon atoms) we have found $\alpha=5$ for $\xi=0.1$ and $\alpha=1.5$ for $\xi=0.5$. 


\section{Clusters illuminated by a laser pulse}

Ground state properties of small clusters of rare-gas atoms have been investigated extensively in the literature (see, e.g., [12-15]). The usual theoretical approach is to describe the atoms in a cluster as a system of particles interacting via a short-range potential such as, e.g., the Lennard-Jones potential. It has been reported that particularly stable configurations of such clusters are of the form of closed-shell icosahedron. Particularly stable structures arise for $N=13$ atoms (one icosahedral shell and one atom in the middle), and $N=55$ (two icosahedral shells and one atom in the middle; the second shell consists of two sub-shells with different radiuses).

We downloaded from [16] the publicly available ground state structures of $N=13$ and $N=55$ atom Lennard-Jones clusters and used them as the equilibrium positions of the nuclei in the Thomas-Fermi model. The positions of the nuclei were rescaled so that equilibrium distance between two atoms is $R_{0} \approx 7$ a.u. $=3.7 \AA$ which is similar to the spacing of atoms in argon clusters [17]. Next, they were used as an input into the hydrodynamic calculations involving the motion of an electron fluid. The resulting example ground-state structures are shown in Figs. 1 and 2. Let us note that in Fig. 1 the electron clouds of individual atoms are well separated. On the contrary, in Fig. 2 we observe the larger radius of the pseudo-particles as compared to Fig. 1. This allows us to model the electron cloud in between the atoms with greater accuracy.

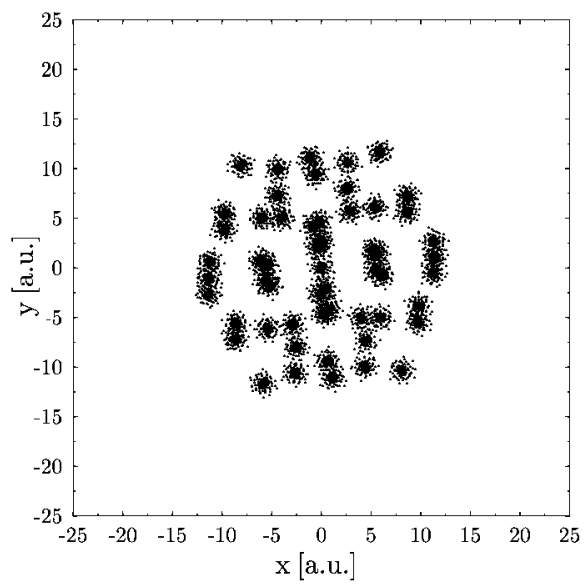

Fig. 1. Ground-state structure of an $N=55$ atom cluster with $\xi=0.1$. Equilibrium positions of the nuclei $\left\{\boldsymbol{R}_{a}\right\}$ are marked by filled circles. Small black dots correspond to the positions of the pseudo-particles modeling the equilibrium electronic density $\rho$.

Let us notice that, as shown by [18], the ground state of a system of interacting Thomas-Fermi atoms corresponds to the situation in which all the internuclear separations are infinite. Thus additional corrections are needed to the 


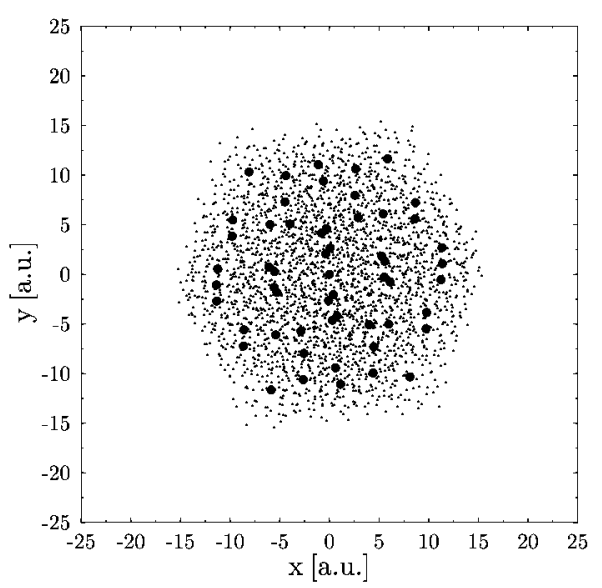

Fig. 2. Ground-state structure of a $N=55$ atom cluster with $\xi=0.5$. Equilibrium positions of the nuclei $\left\{\boldsymbol{R}_{a}\right\}$ are marked by filled circles. Small black dots correspond to the positions of the pseudo-particles modeling the equilibrium electronic density $\rho$.

Thomas-Fermi functional in order to model interatomic bonds. However, it turned out that in the case of a cluster illuminated by a short 100 femtosecond pulse considered in this paper, this restriction does not pose any problems. The ground-state structure of a cluster obtained by a minimization of the standard Thomas-Fermi functional turned out to be stable enough during the pulse.

In the next step we make use of the four obtained ground states (two sizes $N=13$ and $N=55$ as well as two bound electrons ratios $\xi=0.1$ and $\xi=0.5$ ) to proceed with the simulations. We are concerned with the interaction of the

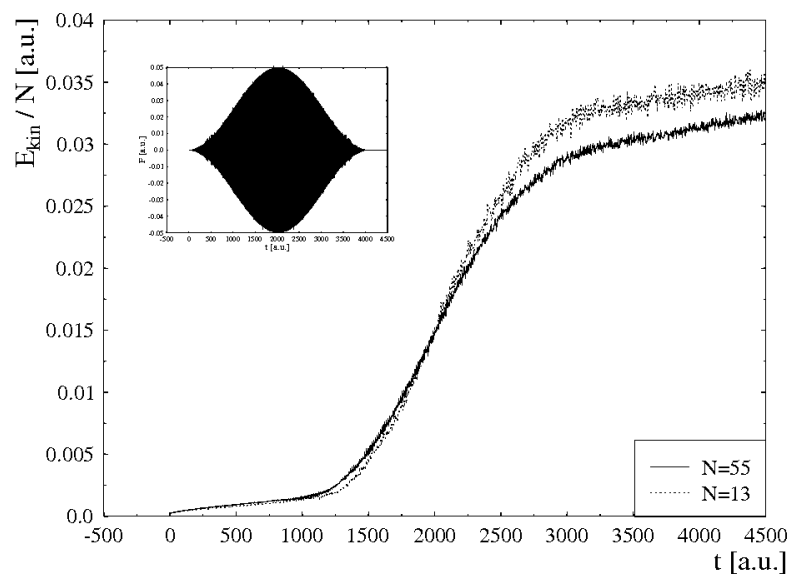

Fig. 3. Kinetic energy of the electrons per atom in a 13 and 55 atom clusters with $\xi=0.1$ illuminated by a free-electron laser pulse plotted versus time. An inset shows the shape of the pulse used in the simulations. 


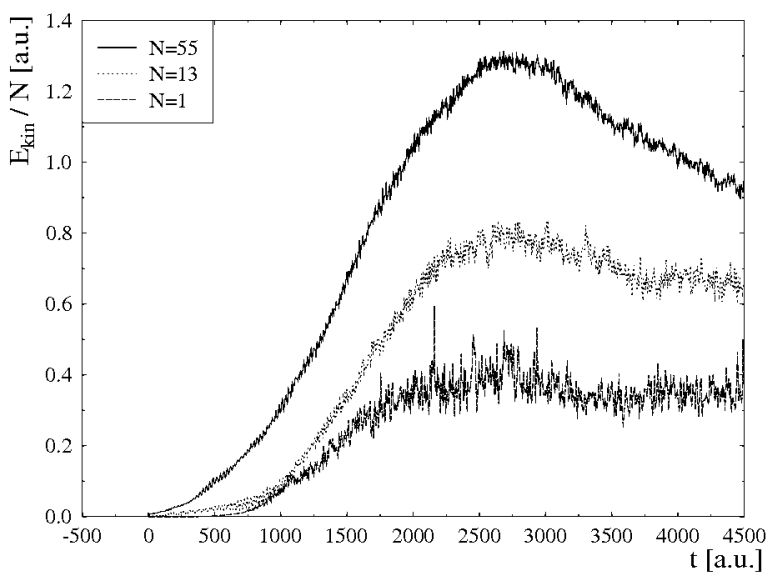

Fig. 4. Kinetic energy of the electrons per atom in a 1, 13, and 55 atom cluster with $\xi=0.5$ illuminated by a free-electron laser pulse plotted versus time.

laser pulse with these clusters. Clusters are subjected to the pulse described by Eq. (7). It turns out that the structures corresponding to $\xi=0.1$ survive the pulse intact - no ions appear and, therefore, no essential motion of nuclei is observed. The only parameter affected by the pulse is kinetic energy of electrons, which in our model is a sum of the kinetic energies of pseudo-particles. It is plotted as a function of time in Figs. 3 and 4.

From Fig. 3 it is seen that for $\xi=0.1$ almost no laser energy is absorbed (about $10 \%$ of a photon per atom). There is no difference in absorption by clusters of different size either. This means that the atoms in the clusters behave more like individual atoms than as a collective system.

This should be contrasted with Fig. 4. Clearly for $\xi=0.5$ the energy absorbed grows with the size of the cluster and corresponds to about 3 photons per atom in the case of a 55 atom cluster. The decrease in the energy after the maximum of the pulse corresponds to the destruction of collective electron oscillations by the cluster explosion.

\section{Ionization and Coulomb explosion}

In Fig. 5 we present the structure of the cluster just after the pulse is finished. It shows that the atoms from the outer shells started to move. Still the atoms of the cluster stay together - the pulse itself was not able to totally disintegrate the cluster which is the case for optical frequences. Although the electron cloud excited by the pulse is still well within the spatial structure of the cluster it already starts to affect the atoms forming the inner shells of the cluster. This could lead in principle to collisional ionization. That it is indeed the case will be seen in the forthcoming figures. The size of the cluster is about twice larger than the ground state structure from Fig. 2. The inset to Fig. 5 illustrates an isotropic character 


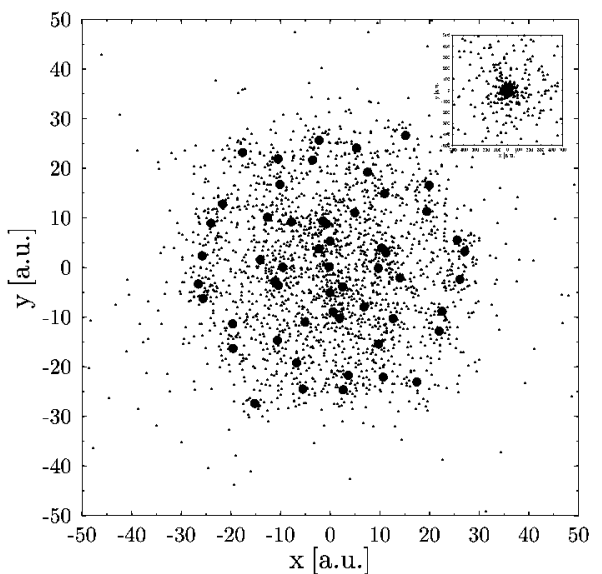

Fig. 5. Excited $N=55$ atom cluster with $\xi=0.5$ at the end of the free-electron laser pulse at $t=4500$ a.u. Positions of the nuclei $\left\{\boldsymbol{R}_{a}\right\}$ are marked by filled circles. Small black dots correspond to the positions of the pseudo-particles modeling the electronic density $\rho$. An inset shows the electron cloud at a 1:10 magnification (i.e., the $x$ and $y$ coordinates vary from -500 to 500 a.u.).

of the explosion (let us remember that the electric field of the laser pulse was polarized along the $x$ axis).

In Fig. 6 we see the cluster structure after longer time, namely twice as long as in the previous case. The cluster behavior is thus influenced by the period of "without-the-pulse" evolution. The outer shell is apparently moving away from

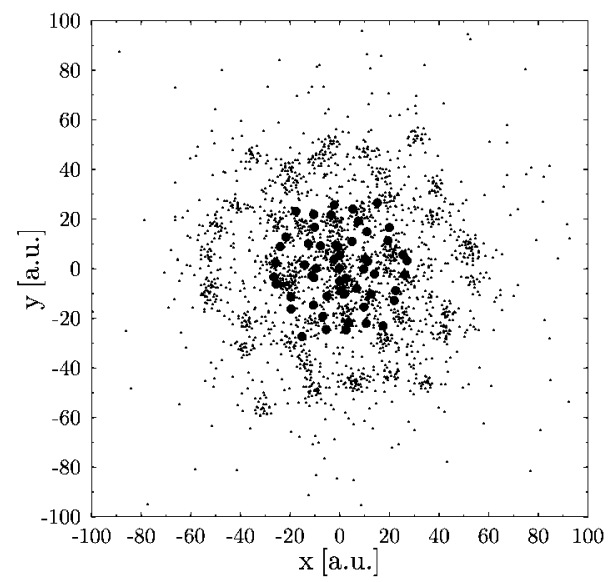

Fig. 6. Excited $N=55$ atom cluster with $\xi=0.5$ at the end of the free-electron laser pulse at $t=9000 \mathrm{a} . u$. The size of the cluster is again about twice larger than the excited state structure from Fig. 5. 
the cluster but the atoms from the deeper located shells are still close to each other. They continue, however, to be ionized by the excited electrons.

To estimate the rate of that electron-driven ionization we plot the average charge of atoms coming from different shells of the cluster. It is depicted in Figs. 7 and Fig. 8. The timescale in these figures is even longer and equal to the duration of the pulse multiplied by factor three. We see that there is life long after the pulse is gone.

From Fig. 8 it is clearly seen that the pulse itself rather excites and not directly ionizes the cluster. Apparently there is a room for the collision-driven

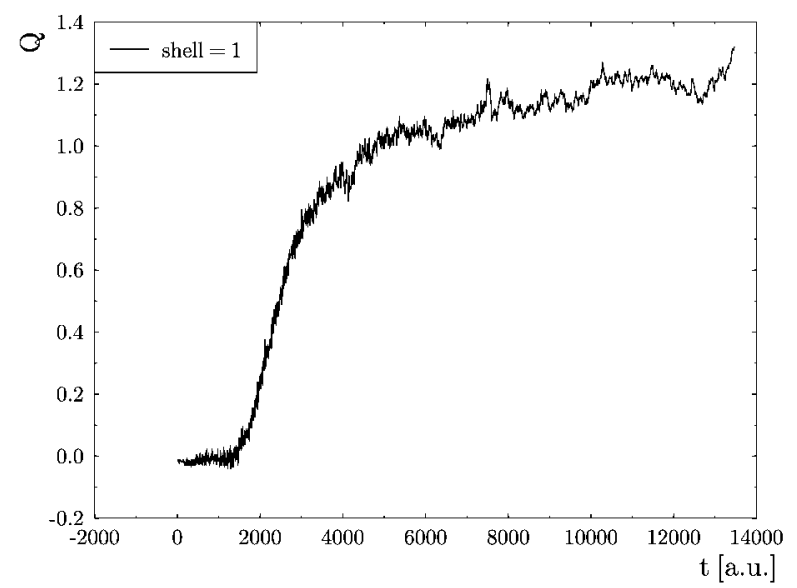

Fig. 7. Average charge $\mathcal{Q}$ of the ions after explosion of an $N=13$ atom cluster versus time $t$. The solid line corresponds to the ions coming from the first shell of the cluster.

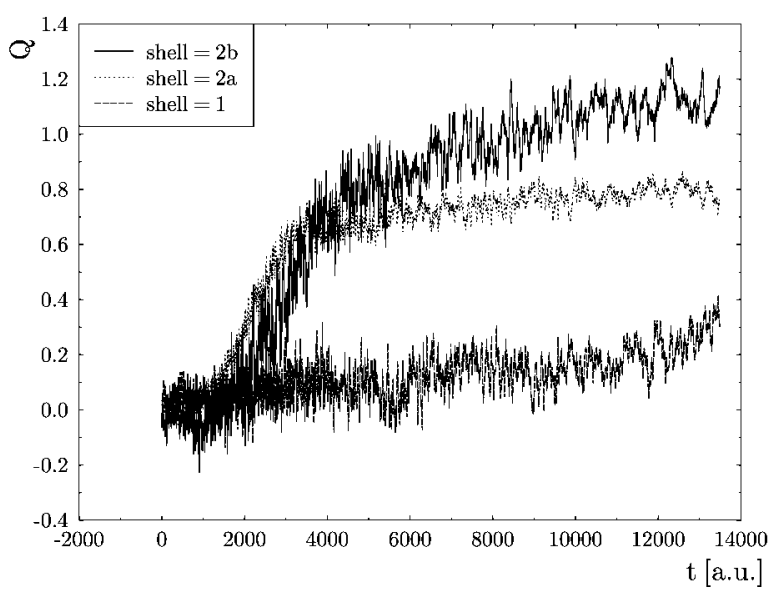

Fig. 8. Average charge $\mathcal{Q}$ of the ions coming from different (sub)shells of an $N=55$ atom cluster from Fig. 2 versus time $t$. 
ionization. Especially the outer shell is armless against the collision ionization caused by all the electrons leaving the cluster (as they have to pass through). There is also an interesting feature of the inner shell behavior. We observe the reasonably rapid increase in the charge at the end of the recorded time. It is definitely not an artifact. This behavior is confirmed by our simulations devoted to long timescale analysis of the cluster explosions, which will be presented in the forthcoming paper.

The charges obtained are indispensable to study the Coulomb explosion processes of the cluster. We model the cluster as a system of point charges with time-dependent values taken from the above-described Thomas-Fermi simulations.

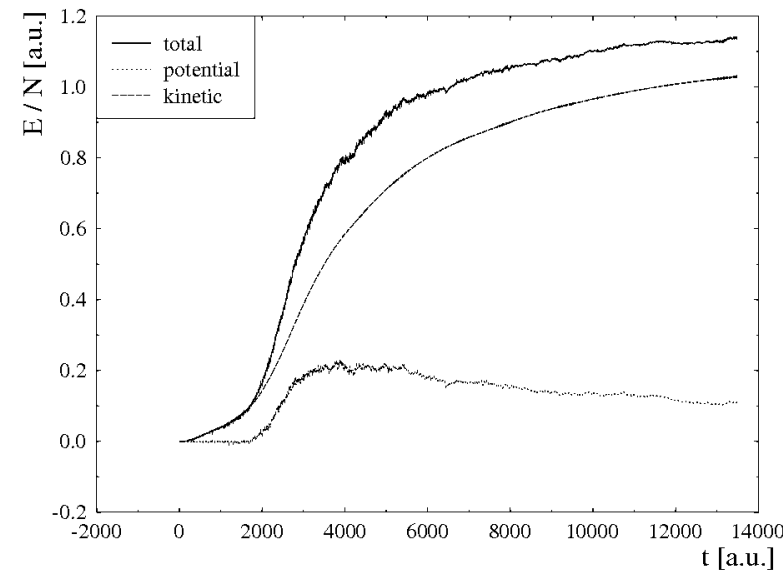

Fig. 9. Kinetic, potential (Coulomb), and total energy of the ions formed in the explosion of the $N=13$ atom cluster from Fig. 7 plotted versus time.

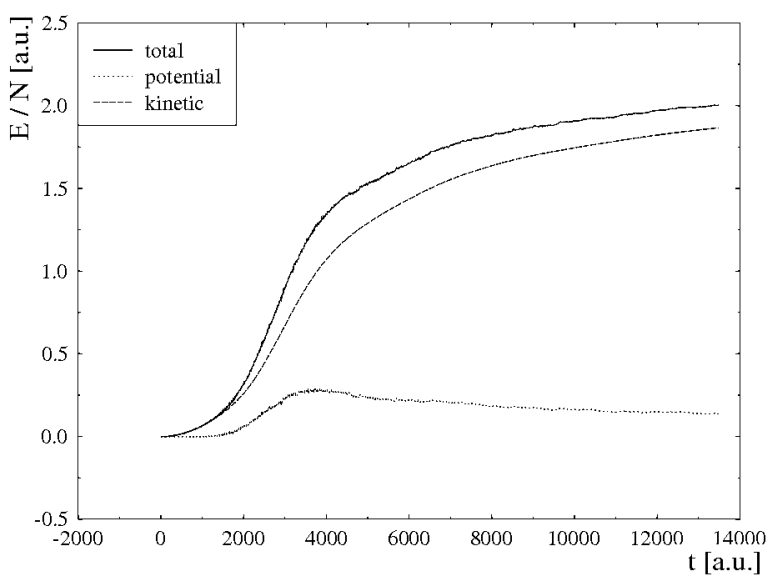

Fig. 10. Kinetic, potential (Coulomb), and total energy of the ions formed in the explosion of the $N=55$ atom cluster from Fig. 8 plotted versus time. The total energy is about twice larger than in Fig. 9. 
These charges are placed at the nuclei positions which enables us to calculate the energy of the Coulomb interactions between the ions and to estimate their final kinetic energy.

In Figs. 9 and 10 we present the kinetic, potential, as well as total energy of these charged particles modeling the ions. It is interesting that the kinetic energy grows not only at the cost of diminishing potential energy but faster than that. This energy excess can be attributed to the additional ionization caused by the excited electron cloud.

The last remaining stage to be done is to estimate the total energy released from the cluster by the pulse. It can be reliably estimated as a sum of kinetic energy of ions and electrons. Results of this analysis are presented below.

As seen from inspection of Figs. 4 and 10 the total energy absorbed by a 55 atom cluster is about 10 photons per atom (3.2 a.u.). Moreover, as follows from comparison of Figs. 9 and 10, the absorbed energy increased with the size of the cluster (about twice when the radius increased also twice). Thus our results may be considered close to the experimental result of 30 photons per atom.

\section{Brief summary}

Using a rather simplified yet powerful approach we have addressed a "hot" topic in the field of cluster physics, namely, that of the rare-gas atomic clusters explosion driven by a short X-ray pulse absorption. Despite its simplicity our time-dependent Thomas-Fermi model happened to provide quite realistic description of both the ionization and explosion processes. Using the pulse parameters of the actual experiments we have obtained quite impressive qualitative agreement with the measured quantities. In particular, we managed to differ from experimental results only by factor of four, which taking into account the modest resources employed, seems to us quite impressive. Let us emphasize that other more advanced theoretical approaches provide much poorer agreement with experimental results (by the factor of 10). In our model the absorbed energy grows with the size of the cluster. This means that by studying larger cluster we can hope to match the experimental results exactly.

\section{Acknowledgment}

This work was supported by the State Committee for Scientific Research under grant No. 5 P03B 06320.

\section{References}

[1] H. Wabnitz, L. Bittner, A.R.B. de Castro, R. Dohrmann, P. Gurtler, T. Laarmann, W. Laasch, J. Schulz, A. Swiderski, K. von Haeften, T. Moller, B. Faatz, A. Fateev, J. Feldhaus, C. Gerth, U. Hahn, E. Saldin, E. Schneidmiller, K. Sytchev, K. Tiedtke, R. Treusch, M. Yurkov, Nature 420, 482 (2002). 
[2] M. Rusek, H. Lagadec, T. Blenski, Phys. Rev. A 63, 013203 (2001).

[3] L.H. Thomas, Proc. Camb. Phil. Soc. 23, 542 (1926).

[4] E. Fermi, Z. Phys. 48, 73 (1928).

[5] L.B. Lucy, Astron. J. 82, 1013 (1977).

[6] R.A. Gingold, J.J. Monaghan, Mon. Not. R. Astr. Soc. 181, 375 (1977).

[7] F. Bloch, Z. Phys. 81, 363 (1933).

[8] J.A. Ball, J.A. Wheeler, E.L. Fireman, Rev. Mod. Phys. 45, 333 (1973).

[9] K. Ishikawa, B.U. Felderhof, T. Blenski, B. Cichocki, J. Plasma Phys. 60, 787 (1998).

[10] P. Gombas, Die Statistische Theorie des Atoms und ihre Anwendungen, Springer, Wien 1949.

[11] N.H. March, Theory of the Inhomogeneous Electron Gas, Eds. S. Lundqvist, N.H. March, Plenum Press, New York 1983, p. 1.

[12] J. Farges, M.F. de Faraudy, B. Raoult, G. Torchet, J. Chem. Phys. 78, 5067 (1983).

[13] J. Farges, M.F. de Faraudy, B. Raoult, G. Torchet, J. Chem. Phys. 84, 3491 (1986)

[14] J.A. Northby, J. Chem. Phys. 87, 6166 (1987).

[15] D.J. Wales, J.P.K. Doye, J. Phys. Chem. A 101, 5111 (1997).

[16] http://www.wales.ch.cam.ac.uk/ jon/structures/L.J.html.

[17] E.D. Potter, Q. Liu, A.H. Zewail, Chem. Phys. Lett. 200, 605 (1992).

[18] E. Teller, Rev. Mod. Phys. 34, 627 (1962). 